\title{
DIFFERENTIABILITY A.E. AND APPROXIMATE DIFFERENTIABILITY A.E.
}

\author{
A. M. BRUCKNER ${ }^{1}$
}

\begin{abstract}
Let $F$ be a finite real valued function defined on $[0,1]$. We prove that $F$ can be transformed into a function which is differentiable a.e. by a homeomorphic change of variables if and only if $F$ is continuous on a dense set. We also show that $F$ can be transformed into a function which is approximately differentiable a.e. if and only if each interval $I \subset[0,1]$ contains a nonempty perfect set $P$ such that $F \mid P$ is continuous.
\end{abstract}

1. Introduction. Recently, Fleissner and Foran [4] have determined that a function $F$ defined on the interval $[0,1]$ can be transformed into a differentiable function by a homeomorphic change of variables if and only if $F$ is continuous and of generalized bounded variation in the restricted sense $\left(\mathrm{VBG}_{*}\right)$. This result complements results obtained in [3], where conditions on $F$ are given for $F$ to be transformable into a function with a bounded derivative or with a continuous derivative. In this paper we investigate the analogous problems for differentiability a.e. and for approximate differentiability a.e. In $\$ 2$ below we characterize the functions which can be transformed into ones which are differentiable a.e. as those which are continuous on a dense subset of $[0,1]$. Thus, even if a continuous function is nowhere differentiable, it can be transformed into one which is differentiable a.e.

In $\$ 3$ we show that the requirement that a function be transformable into one which is approximately differentiable a.e. imposes little restriction on the function. In particular, each measurable function $F$ can be transformed in this way, even if $F$ itself is nowhere approximately differentiable.

In the sequel, we shall make frequent use of notions related to the notion of bounded variation. We refer the reader to Chapter VII of Saks [7] for the necessary definitions. Throughout the article we shall assume all functions are real valued (finite) and we shall denote Lebesgue measure by $\lambda$.

2. Differentiability a.e. Our aim in this section is to prove the following theorem.

THEOREM 1. Let $F$ be a function defined on $[0,1]$. A necessary and sufficient condition for there to exist a homeomorphism $h$ of $[0,1]$ onto itself such that

Received by the editors February 28, 1977.

AMS (MOS) subject classifications (1970). Primary 26A24; Secondary 26A21, 26A45.

Key words and phrases. Differentiability, approximate differentiability, generalized bounded variation (VBG, VBG.

1 The author was supported in part by NSF grant MCS 76-06573.

(c) American Mathematical Society 1977 
$F \circ h$ is differentiable a.e. is that $F$ be continuous on a dense subset of $[0,1]$.

To simplify the proof of Theorem 1, we begin with three lemmas.

LEMMA 1. A function $F$ defined on $[0,1]$ is differentiable a.e. if and only if there is a set $Z$ of measure zero such that $F$ is $\mathrm{VBG}_{*}$ on $[0,1] \sim Z$.

Proof. This lemma is essentially contained in Saks [7]: if $F$ is $\mathrm{VBG}_{*}$ on a set $E$, then $F$ is differentiable a.e. on $E$ (p. 230), and if $F$ is differentiable on a set $E$ then $F$ is $\mathrm{VBG}_{*}$ on $E$ (p. 234).

We mention that another criterion for differentiability a.e. has been advanced by Pettineo [6], but we shall not need that criterion in this paper.

LEMMa 2. Let $Q$ be a first category set of type $F_{\sigma}$ which is c-dense in $[0,1]$. Then there exists a homeomorphism $h$ of $[0,1]$ onto itself such that $\lambda(h(Q))=1$.

Lemma 2 is just a restatement of Lemma 5 of Gorman [5].

LEMMA 3. Let $F$ be defined on $[0,1]$ and let $P$ be a nonempty nowhere dense perfect subset of $[0,1]$. If $F$ is continuous at each point of $P$, then there exists a nonempty perfect set $Q \subset P$ such that $F$ is $\mathrm{VB}_{*}$ on $Q$.

Proof. For each $x$ and $y \in[0,1]$ let $\omega(x, y)$ denote the oscillation of $F$ on $[x, y]$ : i.e., $\omega(x, y)=\sup \{|F(s)-F(t)|: s, t \in[x, y]\}$. Since $F$ is continuous on $P$, and $P$ is compact, there exists a sequence of numbers $\left\{\delta_{n}\right\}$ decreasing to 0 such that $\omega(x, y)<1 / 3^{2 n}$ whenever $x \in P, y \in[0,1]$ and $|x-y|<\delta_{n}$.

We shall define a countable set $D$ contained in $P$, such that $D$ is dense-initself, and such that $F$ is $\mathrm{VB}_{*}$ on $D$. The set $Q=\bar{D}$ is then a perfect subset of $P$ on which $F$ is $\mathrm{VB}_{*}$.

In order to avoid notation involving multiple subscripts, we shall introduce special language and notation as we proceed through the construction of $D$. Choose an interval $[a, b]$ of length less than $\delta_{1}$ such that $a$ and $b$ are bilateral points of accumulation of $P$ and call this interval an interval of rank 1. Now choose two intervals $[c, d]$ and $[e, f]$ such that $c, d, e$ and $f$ are bilateral points of accumulation of $P$ which satisfy the inequalities $c<d<a<b<e<f$, $a-c<\delta_{2}$ and $f-b<\delta_{2}$. We call [c,d] and $[e, f]$ intervals of rank two. We proceed inductively-if we already have determined the $3^{n-1}$ intervals of rank $n$ or less we choose $2 \cdot 3^{n-1}$ intervals of rank $n+1$ in such a way as to satisfy the following conditions:

(i) Each such interval has endpoints which are bilateral points of accumulation of $P$.

(ii) To each interval $[p, q]$ of rank $n$ or less, correspond two intervals $[r, s]$ and $[t, u]$ of rank $n+1$ such that $r<s<p<q<t<u, p-r<\delta_{n+1}$ and $u-q<\delta_{n+1}$.

(iii) The resulting $3^{n}$ intervals of rank no greater than $n+1$ are pairwise disjoint.

It is easy to verify that such a construction is possible-the possibility of satisfying conditions (ii) and (iii) for the intervals of rank $n+1$ follows from 
condition (i) for the intervals of rank $n$ or less.

For each integer $n$, let $D_{n}$ be the set of endpoints of intervals of rank $n$ and let $D=\cup_{n=1}^{\infty} D_{n}$. It is clear from (ii) that $D$ is dense-in-itself. We show $F$ is $\mathrm{VB}_{*}$ on $D$. Let $n$ be a positive integer and let $d_{1}<d_{2}<\cdots<d_{3^{n}}$ be the points of $D_{1} \cup D_{2} \cup \cdots \cup D_{n}$. Let $\sigma_{n}=\sum_{k=1}^{3^{n}-1} \omega\left(d_{k}, d_{k+1}\right)$. Let $\sigma_{n+1}$ be the corresponding sum for the set $D_{1} \cup D_{2} \cup \cdots \cup D_{n} \cup D_{n+1}$. We show $\sigma_{n+1} \leqslant \sigma_{n}+4 / 3^{n+3}$. To verify this inequality, observe first that the addition of the points of $D_{n+1}$ results in three changes in the computation of $\sigma_{n}$.

(a) There are $2 \cdot 3^{n-1}$ intervals of rank $n+1$. By (ii), each of these is of length less than $\delta_{n+1}$. Thus, by the definition of the number $\delta_{n+1}$, the total contribution of the intervals to the sum $\sigma_{n+1}$ does not exceed $2 \cdot 3^{n-1} / 3^{2 n+2}$ $=2 / 3^{n+3}$.

(b) There are also $2 \cdot 3^{n-1}$ intervals with one endpoint in $D_{n+1}$ and the other in $D_{n}$. Each of these intervals is also of length less than $\delta_{n+1}$ because of (ii), thus their total contribution to the sum $\sigma_{n+1}$ does not exceed $2 / 3^{n+3}$.

(c) Each remaining interval $(a, b)$ contributing to the sum $\sigma_{n+1}$ either is an interval of rank $n$, say $\left(d_{k}, d_{k+1}\right), k$ odd, or is contained in an interval $\left(d_{k}, d_{k+1}\right), k$ even. In the first case, the number $\omega\left(d_{k}, d_{k+1}\right)$ has already been computed as part of the sum defining $\sigma_{n}$. In the second case, $\omega(a, b) \leqslant$ $\omega\left(d_{k}, d_{k+1}\right)$. Since only one such interval $(a, b)$ can be contained in $\left(d_{k}, d_{k+1}\right)$ for each $k$, the total contribution of all such intervals to the sum $\sigma_{n+1}$ cannot exceed $\sigma_{n}$.

Combining the estimates from (a), (b) and (c), we infer $\sigma_{n+1} \leqslant \sigma_{n}+$ $4 / 3^{n+3}$. Since $\sigma_{1} \leqslant \frac{1}{9}$, we see $\sigma_{n+1} \leqslant \frac{1}{9}+\sum_{k=1}^{n} 4 / 3^{k+3}$. It is now easy to see that $F$ is $\mathrm{VB}_{*}$ on $D$. If $x_{1}<x_{2}<\cdots<x_{m}$ are points in $D$, there exists a positive integer $n$ such that $x_{k} \in D_{1} \cup \cdots \cup D_{n}$ for all $k=1, \ldots, m$. Thus

$$
\sum_{k=1}^{m-1} \omega\left(x_{k}, x_{k+1}\right) \leqslant \sigma_{n} \leqslant \frac{1}{9}+\sum_{k=1}^{n-1} \frac{4}{3^{k+3}}<\frac{1}{9}+\sum_{k=1}^{\infty} \frac{4}{3^{k+3}}<\infty,
$$

and $F$ is $\mathrm{VB}_{*}$ on $D$. Let $Q=\bar{D}$. Then $Q \subset P$. The set $Q$ is perfect because $D$ is dense-in-itself and $F$ is $\mathrm{VB}_{*}$ on $Q$. Thus the proof of Lemma 3 is complete.

Proof OF THEOREM 1. If $F$ is discontinuous at each point of some interval $I$ and $h$ is a homeomorphism of $[0,1]$ onto itself, then $F \circ h$ is discontinuous at each point of the interval $h^{-1}(I)$, so $F \circ h$ cannot be differentiable a.e. Thus, the condition is necessary.

To prove the sufficiency, we observe that the hypothesis implies $F$ is continuous on a set $A$ of type $G_{\delta}$ and dense in $[0,1]$. Let $\left\{I_{n}\right\}$ be an enumeration of the intervals with rational endpoints contained in $[0,1]$. Now every dense set of type $G_{\delta}$ contains a nonempty perfect set. Thus, to each interval $I_{n}$ corresponds a nonempty nowhere dense perfect subset $P_{n}$ of $I_{n} \cap A$. By Lemma 3 , there exists a nonempty perfect subset $Q_{n}$ of $P_{n}$ such that $F$ is $\mathrm{VB}_{*}$ on $Q_{n}$. Let $Q=\cup_{n=1}^{\infty} Q_{n}$. Then $Q$ is of type $F_{\sigma}$ and is a $c$-dense subset of $[0,1]$. Furthermore, $F$ is $\mathrm{VBG}_{*}$ on $Q$. By Lemma 2, there exists a homeomorphsim $h$ of $[0,1]$ onto itself such that $\lambda(h(Q))=1$. The function 
$F \circ h^{-1}$ is $\mathrm{VBG}_{*}$ on $h(Q)$. Thus, by Lemma $1, F \circ h^{-1}$ is differentiable a.e. on $h(Q)$, and the proof of Theorem 1 is complete.

COROLLARY. Every function in the first class of Baire can be transformed into a function which is differentiable a.e. by a homeomorphic change of variables.

This is so because each such function is continuous on a dense set.

We cannot replace Baire class 1 by Baire class 2 in the enunciation of the corollary, because Baire 2 functions can be discontinuous everywhere.

For continuous functions, the corollary was established by Bari [1].

3. Approximate differentiability a.e. We turn now to the problem of creating approximate differentiability a.e. Since approximate differentiability is a much weaker condition than differentiability, we might expect there to be a very large class of functions which are transformable into ones which are approximately differentiable a.e. This turns out to be the case (in spite of the fact that even a continuous function might be approximately differentiable almost nowhere (Saks [7, p. 297])).

TheOREM 2. Let $F$ be a function defined on $[0,1]$. A necessary and sufficient condition for there to exist a homeomorphism $h$ of $[0,1]$ onto itself such that $F \circ h$ is approximately differentiable a.e. is that to each interval $I \subset[0,1]$ corresponds a nonempty perfect set $P \subset I$ such that $F \mid P$ is continuous.

Proof. The necessity of the condition is clear: by Luzin's theorem $F \circ h$ must satisfy it, so $F$ must also. We turn therefore to a proof of the sufficiency of the condition. Suppose $F$ satisfies the condition. Let $\left\{I_{n}\right\}$ be an enumeration of those subintervals of $[0,1]$ which have rational endpoints. To each such interval corresponds a nonempty nowhere dense perfect set $P_{n}$ such that $F \mid P_{n}$ is continuous. By Theorem 3.2 of [2], there exists, for each $n$, a nonempty perfect set $Q_{n}$ contained in $P_{n}$ such that $F \mid Q_{n}$ has a derivative, finite or infinite, at each point of $Q_{n}$. (Difference-quotients are computed only for points of $Q_{n}$ here.) Thus $F$ is VBG on $Q_{n}$ [7, p. 237]. Let $Q=\cup Q_{n}$. Then $Q$ is of type $F_{\sigma}, Q$ is $c$-dense in $[0,1]$ and the function $F$ is VBG on $Q$. Using Lemma 2, we obtain a homeomorphism $h$ of $[0,1]$ onto itself such that $\lambda(h(Q))=1$. It is clear that $F \circ h^{-1}$ is VBG on $h(Q)$. Since for each $n, F \mid Q_{n}$ is continuous, $F \circ h^{-1}$ is measurable on $h(Q)$, and since $\lambda(h(Q))=1$, $F \circ h^{-1}$ is measurable on all of $[0,1]$. Therefore $\left[7\right.$, p. 227] $F \circ h^{-1}$ is approximately differentiable a.e. on $h(Q)$ and the proof of Theorem 2 is complete.

COROLlary. If $F$ is either measurable or possesses the Property of Baire, then $F$ can be transformed into a function which is approximately differentiable a.e. by a homeomorphic change of variables.

Proof. For measurable functions, the result follows from Luzin's theorem. If $F$ possesses the Property of Baire, then there is a residual set $E$ such that 
$F \mid E$ is continuous. Each residual set contains nonempty perfect sets in every interval, so the result follows.

The result follows equally for any function measurable with respect to some nonatomic Lebesgue-Stieltjes measure $\mu$ such that $\mu(I)>0$ for every interval $I \subset[0,1]$. There are, of course, functions which cannot be transformed into ones which are approximately differentiable a.e. For example, if $F$ takes on every real value on every nonempty perfect set, and $h$ is any homeomorphism of $[0,1]$ onto itself, then $F \circ h$ cannot be measurable (it violates Luzin's theorem), so $F \circ h$ cannot be approximately differentiable a.e.

We can compare the hypotheses of the two theorems. The condition of Theorem 1 is equivalent to the condition that to each interval $I \subset[0,1]$ corresponds a nonempty perfect set $P$ such that $F$ is "genuinely" continuous on $P$ whereas the condition of Theorem 2 requires only that the restriction $F \mid P$ of $F$ to $P$ be continuous.

As a final remark, we relate Theorem 2 to a theorem of Whitney [8]. This theorem asserts that a function $F$ can be approximated in the Luzin sense by a continuously differentiable function if and only if $F$ is approximately differentiable a.e. Thus, Theorem 2 can be interpreted as stating that every function meeting the hypothesis of the theorem can be transformed into one which is equal to a continuously differentiable function except on a set of arbitrarily small measure.

\section{REFERENCES}

1. N. Bari, Mémoire sur la représentation finie des fonctions continue, Math. Ann. 103 (1930), 185-248; 598-653.

2. A. M. Bruckner, J. Ceder and M. Weiss, On the differentiability structure of real functions, Trans. Amer. Math. Soc. 142 (1969), 1-13.

3. A. M. Bruckner and C. Goffman, Differentiability through change of variables, Proc. Amer. Math. Soc. 61 (1976), 235-241.

4. R. Fleissner and J. Foran, Letter to the author, 1976.

5. W. Gorman, The homeomorphic transformation of c-sets into d-sets, Proc. Amer. Math. Soc. 17 (1966), 825-830.

6. B. Pettineo, Quelques observations sur les fonctions dérivables presque partout, C. R. Acad. Sci. Paris 248 (1959), 518-520.

7. S. Saks, Theory of the integral, Monographie Mat., tom 7, Warsaw, 1937.

8. H. Whitney, On totally differentiable and smooth functions, Pacific J. Math. 1 (1951), 143-159.

Department of Mathematics, University of California, Santa barbara, California 93106 